\title{
Spin-valve effect in a carbon atomic wire
}

\author{
Yadong Wei, ${ }^{1}$ Yan $\mathrm{Xu},{ }^{2}$ Jian Wang, ${ }^{2,3, *}$ and Hong Guo ${ }^{4}$ \\ ${ }^{1}$ Department of Physics, College of Science, Shenzhen University, Shenzhen, P.R. China \\ ${ }^{2}$ Department of Physics, The University of Hong Kong, Pokfulam Road, Hong Kong, China \\ ${ }^{3}$ Key Laboratory of Materials Physics, Institute of Solid State Physics, Chinese Academy of Sciences, Hefei, Anhui, P. R. China \\ ${ }^{4}$ Department of Physics, McGill University, Montreal, PQ, H3A, 2T8, Canada
}

(Received 2 July 2004; revised manuscript received 26 August 2004; published 16 November 2004)

\begin{abstract}
We report a theoretical investigation of the spin-valve effect in an atomic scale system, a carbon chain, generated by the presence of a magnetic field in the device leads. We found that there exists a cutoff energy beyond which the conductance of the device vanishes. This cutoff energy can be critically controlled by the relative orientation of the magnetic fields applied to the leads, so that an atomic scale spin valve can be achieved that switches off electric current when magnetic fields of left and right leads are anti-parallel. The physical origin of this transport behavior is found to be related to the wave-function overlap between the leads and the device scattering region.
\end{abstract}

DOI: 10.1103/PhysRevB.70.193406

PACS number(s): 85.35.-p, 72.25.-b, 85.65.+h

Spin polarized charge transport in giant magnetoresistance (GMR) and tunnel magnetoresistance (TMR) systems provides an exciting arena for investigating various spinrelated phenomena. ${ }^{1}$ These systems have also found very important practical applications in information technology, ${ }^{1}$ and provided a working example of spintronics. A basic TMR system consists of a tunnel barrier sandwiched between two ferromagnetic (FM) metal leads, and the TMR effect ${ }^{2}$ arises as a result of energy level mismatch in the two FM leads due to the change of relative orientation of their magnetic moments. When this orientation is varied, the tunnel current is tuned accordingly, producing the so called spin-valve effect $^{2,3}$ which has now been achieved at room temperature. ${ }^{4}$ So far, many investigations have contributed to help establishing the understanding on quantum transport through TMR devices. ${ }^{6-18}$

When the tunnel barrier is a molecule, it is possible to achieve TMR at the molecular scale: an example is the carbon nanotube based TMR devices fabricated recently. ${ }^{5}$ Theoretically such a nanotube TMR, has been investigated using a simple tight binding model, ${ }^{12,17}$ but in general there is currently no theory for spin-molecular electronics because the conventional magnetic tunnel junction theory ${ }^{3}$ does not directly incorporate atomistic properties of the constituent material. At the molecular scale, the atomistic details and chemistry play very important roles in determining the transport outcome, ${ }^{19}$ and this should still be true for molecular scale TMR systems. Although tight binding models can capture, to some degree, the microscopic details of a device, a fully self-consistent density functional theory (DFT) analysis is very desirable to reveal the full transport physics of molecular scale TMR. It is the purpose of this paper to report a theory and its numerical results for a model molecular TMR junction.

In particular, we have developed a theoretical formalism suitable for use in first principles DFT calculations including all atomic degrees of freedom. In this theory, the spin-valve or TMR effect is generated by applying uniform magnetic fields $\mathbf{B}$ to the two metal leads. When the magnetic fields on the two leads are either parallel or anti-parallel, very different transport features are obtained leading to the TMR. Experimentally, the local fields in the leads are typically produced by using magnetic materials with different coercivities, ${ }^{1}$ although the physics of the spin-valve effect is the same. ${ }^{2}$ While the theoretical formalism can be applied to many different molecular devices in the form of metalmolecule-metal, as an example, we investigate transport features of a system in which the molecule is a short carbon chain. As studied before, ${ }^{20,21}$ a carbon chain in contact with two metal leads have many interesting transport features including an even-odd conductance behavior ${ }^{20}$ and negative different resistance. ${ }^{21}$ It provides a simple molecular scale conductor for revealing the influence of molecular chemistry to quantum transport physics.

Assuming that the magnetic field on the left lead is pointing to the $z$ direction, the transport direction is in the $y$ direction, while the magnetic field on the right lead is at an angle $\theta$ to the $z$ axis in the $x z$ plane. For quantum transport through atomic scale junctions, the overlap of the Bloch scattering states in the leads and the wave function of the device scattering region plays an important role in determining the transport properties. When the wave functions of the lead and device region are orthogonal to each other, the conductance vanishes. For the device made of a short carbon chain connected by two Al electrodes, we find that there is a cutoff energy beyond which the transmission coefficient is zero. In the presence of a magnetic field, an electron with different spin experiences different potential landscape resulting to a shift of the Fermi level. This in turn shifts the cutoff energy depending on the relative orientation of the magnetic fields of left and right leads. As a result, electric current can be switched off near the cutoff energy when the magnetic fields are anti-parallel. This gives rise to a large TMR ratio of order 1. The TMR due to other mechanisms are also studied and both positive and negative TMR ratios are observed.

We model the atomic device by the following Hamiltonian: 


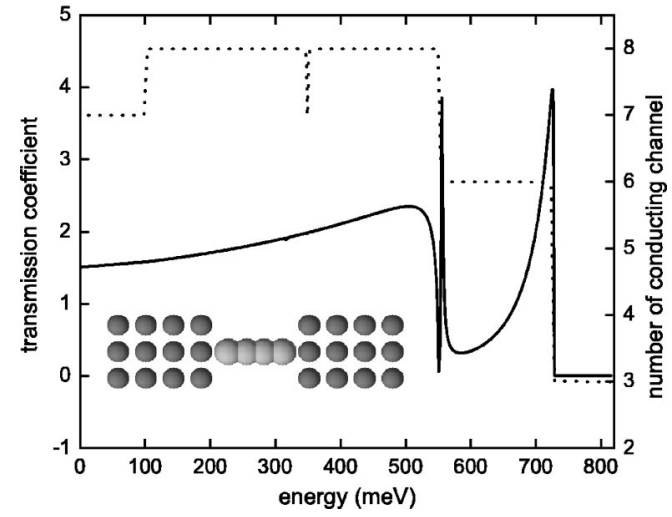

FIG. 1. Transmission coefficient (solid line) and the number of conducting channels (dotted line) in the Al lead vs energy for $\mathrm{Al}-C_{4}-\mathrm{Al}$ junction. Inset: schematic plot of the $\mathrm{Al}-C_{4}$ - $\mathrm{Al}$ junction.

$$
H=H_{L}+H_{R}+H_{0}+H_{T}
$$

where $H_{L}$ and $H_{R}$ describe, respectively, the left and right leads $H_{L}=\Sigma_{k \sigma}\left(\epsilon_{k L}+\sigma M\right) c_{k L \sigma}^{\dagger} c_{k L \sigma}$ and $H_{R}=\Sigma_{k \sigma}\left(\epsilon_{k R}\right.$ $+\sigma M \cos \theta) c_{k R \sigma}^{\dagger} c_{k R \sigma}+\sum_{k \sigma} M \sin \theta\left[c_{k R \sigma}^{\dagger} c_{k R \bar{\sigma}}\right]$. The Hamiltonian $H_{L}$ and $H_{R}$ describe a more general situation in which both leads can be ferromagnetic with magnetization $M$. For nonmagnetic leads in an external magnetic field, we replace $M$ by $\mu \mathbf{B}$, where $\mu$ is the orbital magnetic moment whose value depends on the material. For an electron in an atom, $\mu$ is just the Bohr magneton $\mu_{B}$. In Eq. (1), $H_{0}$ describes the device scattering region, $H_{0}=\sum_{n \sigma} \epsilon_{n} d_{n \sigma}^{\dagger} d_{n \sigma}, H_{T}$ models the coupling between electrodes and the device scattering region with hopping matrix $T_{k \alpha n}$. To simplify the analysis, we assume the hopping matrix to be independent of spin index, hence $H_{T}=\sum_{k \alpha n \sigma}\left[T_{k \alpha n} c_{k \alpha \sigma}^{\dagger} d_{n \sigma}+\right.$ c.c. $]$. In these expressions, $\epsilon_{k \alpha}=\epsilon_{k}^{0}+q V_{\alpha}$ with $\alpha=L, R ; c_{k \alpha \sigma}^{\dagger}$ (with $\sigma=\uparrow, \downarrow$ or \pm 1 and $\bar{\sigma}$ $=-\sigma)$ is the creation operator of electrons with spin index $\sigma$ inside the $\alpha$-electrode. Similarly, $d_{n \sigma}^{\dagger}$ is the creation operator of electrons with spin $\sigma$ at energy level $n$ for the device scattering region. Finally, due to electron-electron interactions, the Hartree potential $U[\rho]$, which is a function of charge density $\rho$, as well as the potential due to exchange and correlation effects $V_{x c}[\rho]$ must be considered. Hence, the actual Hamiltonian of the device scattering region is $H_{0}$ $+q U+q V_{x c}$.

For this system, the electric current can be obtained using the Keldysh nonequilibrium Green's function (NEGF) $\operatorname{approach}^{13}(\hbar=1)$, as

$$
I_{L}=\frac{2 q}{\pi} \int d E \operatorname{Tr}\left[\operatorname{Im}\left(\Sigma_{L}^{r}\right) \mathbf{G}^{r} \operatorname{Im}\left(\Sigma_{R}^{r}\right) \mathbf{G}^{a}\right]\left(f_{L}-f_{R}\right),
$$

where $f_{\alpha} \equiv f\left(E-q V_{\alpha}\right)$ and the trace is over both the state index and spin index. Here $\mathbf{G}^{r}(E, U)$ is the $2 \times 2$ matrix in spin space for the retarded Green's function with $U(\mathbf{r})$ the Hartree potential inside the device scattering region. $\mathbf{G}^{r}(E, U)$ is given by ${ }^{13}$

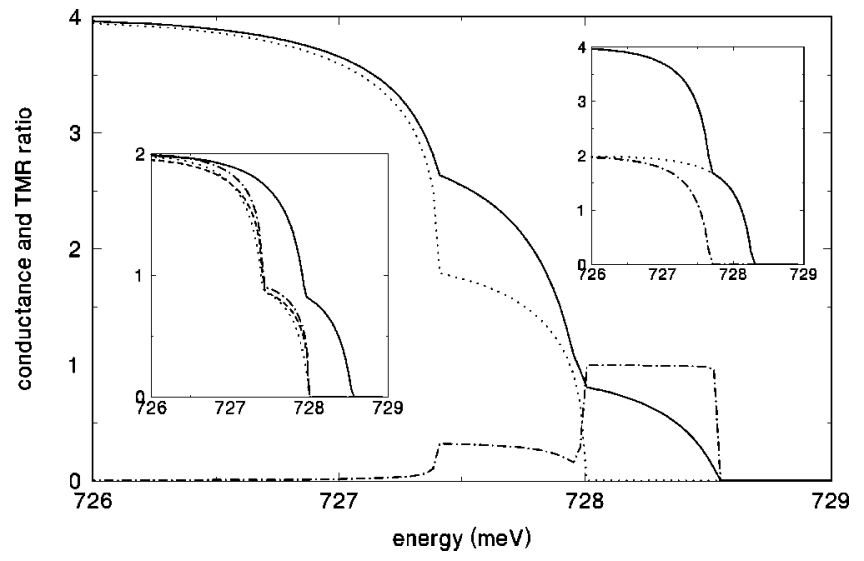

FIG. 2. The conductance $G_{F}$ (solid line), $G_{A}$ (dotted line), and TMR ratio (dotted-dash line) vs energy. The external magnetic field is $5 \mathrm{~T}$ and $\mu=\mu_{B}$. Right inset: the transmission coefficient (solid line) and its eigen-channels vs energy in the absence of magnetic field. Left inset: the conductances $G_{\uparrow \uparrow}$ (solid line), $G_{\downarrow \downarrow}$ (dotted line), $G_{\uparrow \downarrow}$ (dashed line), and $G_{\downarrow \uparrow}$ (dash-dotted line) vs energy. The unit of conductance used in this figure, Fig. 3, and Fig. 4 is $e^{2} / h$.

$$
\mathbf{G}^{r}(E, U)=1 /\left(E-H_{0}-q U-q V_{x c}-\Sigma^{r}\right),
$$

where the self-energy $\Sigma^{r} \equiv \Sigma_{L}^{r}\left(E-q V_{L}\right)+\Sigma_{R}^{r}\left(E-q V_{R}\right)$ is also a $2 \times 2$ matrix in spin space, which describes the coupling of the device scattering region to the two atomic leads and is written as

$$
\Sigma_{\alpha}^{r}(E)=\hat{R}_{\alpha}\left(\begin{array}{cc}
\Sigma_{\alpha \uparrow}^{r} & 0 \\
0 & \Sigma_{\alpha \downarrow}^{r}
\end{array}\right) \hat{R}_{\alpha}^{\dagger}
$$

with the rotational matrix $\hat{R}_{\alpha}$ for electrode $\alpha$ defined as

$$
\hat{R}_{\alpha}=\left(\begin{array}{cc}
\cos \theta_{\alpha} / 2 & \sin \theta_{\alpha} / 2 \\
-\sin \theta_{\alpha} / 2 & \cos \theta_{\alpha} / 2
\end{array}\right) .
$$

Here angle $\theta_{\alpha}$ is defined as $\theta_{L}=0$ and $\theta_{R}=\theta$. The spin-valve effect is closely related to the magnetic field-dependent selfenergy $\Sigma_{\alpha \sigma}^{r}$ as

$$
\Sigma_{\alpha \sigma m n}^{r}=\sum_{k} T_{k \alpha m}^{*} T_{k \alpha n} /\left(E-\epsilon_{k \alpha}-\sigma M+i 0^{+}\right),
$$

which must be calculated for the semi-infinite atomic electrodes within a first-principles technique.

In Eq. (2) the Hartree potential $U(\mathbf{r})$ is determined by the self-consistent Poisson equation

$$
\nabla^{2} U=-4 \pi \rho=2 i q \int d E \sum_{\sigma}\left(\mathbf{G}^{<}(E, U)\right)_{\sigma \sigma},
$$

where the lesser Green's function $\mathbf{G}^{<}$in Eq. (6) is calculated through the Keldysh equation. Equations (2) and (6) are equivalent to the Kohn-Sham (KS) equation in the scattering case and they completely determine the nonlinear $I-V$ characteristics of any multiprobe atomic TMR junctions.

We solve Eqs. (2) and (6) using the ab initio technique of Ref. 22 where DFT is carried out within the NEGF formalism. In the NEGF-DFT analysis, we use an $s, p, d$ real space linear combination of atomic orbitals (LCAO) basis $\operatorname{set}^{22,23}$ 


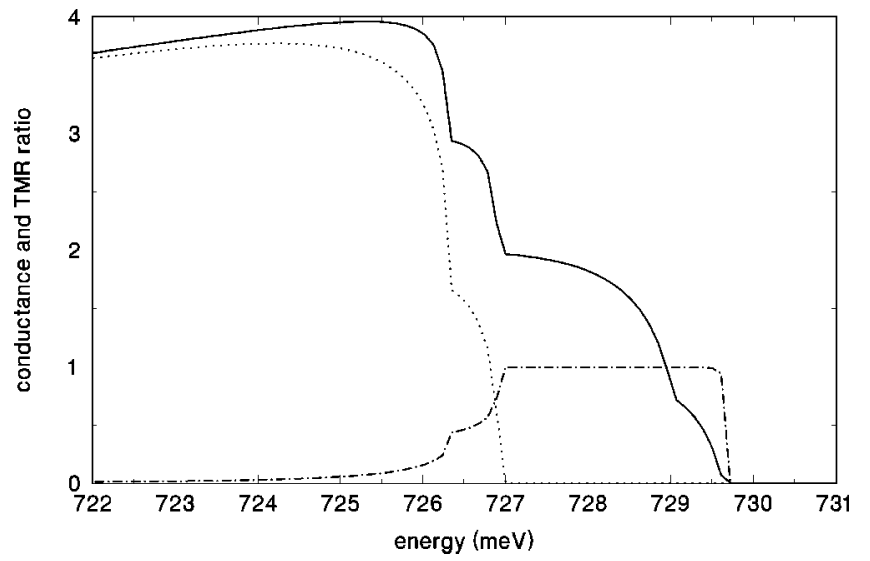

FIG. 3. The conductance $G_{F}$ (solid line), $G_{A}$ (dotted line), and TMR ratio (dotted-dash line) vs energy. The external magnetic field is $5 \mathrm{~T}$ and $\mu=5 \mu_{B}$.

and define atomic cores by standard nonlocal norm conserving pseudopotential. ${ }^{24}$ The density matrix of the device is constructed via NEGF and the semi-infinite leads provide real space potential boundary conditions for the KS potential of the device scattering region: this region consists of the molecule plus several layers of the metal leads. The KS potential includes contributions from Hartree, exchange, correlation, the atomic core, and any other external potentials. The Poisson equation is solved using multigrid method in which the boundary condition is not periodic when the external bias is present. To do that one has to transform from the LCAO basis set used in solving Green's function to a threedimensional grid. This is the most time-consuming part of the $a b$ initio calculation. The NEGF-DFT iteration is numerically converged to $10^{-4} \mathrm{eV}$ which we determine to be reasonable for our purpose. For analyzing the spin-valve effect, the NEGF-DFT formalism produces all the NEGF needed. The NEGF-DFT formalism ${ }^{22,23}$ is now a standard tool for $a b$ initio atomistic calculations of transport and we refer interested readers to the existing literature. ${ }^{22,23}$ For this work, because the field can be different in the two leads, a fielddependent self-energy of the leads Eq. (5), needs to be calculated using a transfer matrix numerical method ${ }^{22}$ for each value of the field strength.

In this work, we focus on the spin-valve effect in the small bias limit. $G_{F}$ and $G_{A}$ denote the conductances of the system when the two magnetic fields $(M=\mu B)$ are parallel and anti-parallel, respectively. The TMR ratio can be defined as $\kappa=\left(G_{F}-G_{A}\right) / G_{F}{ }^{11}$ The system we now consider is a fouratom carbon chain connected by two $\mathrm{Al}$ atomic leads ${ }^{25}$ (see inset of Fig. 1) that has been subjected to study without magnetic fields before. ${ }^{20,26}$ In the calculation, we set $\mu=\mu_{B}$ and the magnetic field to be $B=5 \mathrm{~T}$. We have neglected the effect of magnetic field on the band structure of the system. The transmission coefficient $T(E)$ (including spin factor) as a function of energy is plotted in Fig. 1. We have also shown the number of conducting channels $N_{A l}$ in the Al lead as a function of energy. The variation of $N_{A l}$ reflects the band structure of the $\mathrm{Al}$ lead. We observe that at the Fermi energy $E=0$, the system is fairly transmissive with $T(E)=1.5$. As one increases the energy, the transmission coefficient also

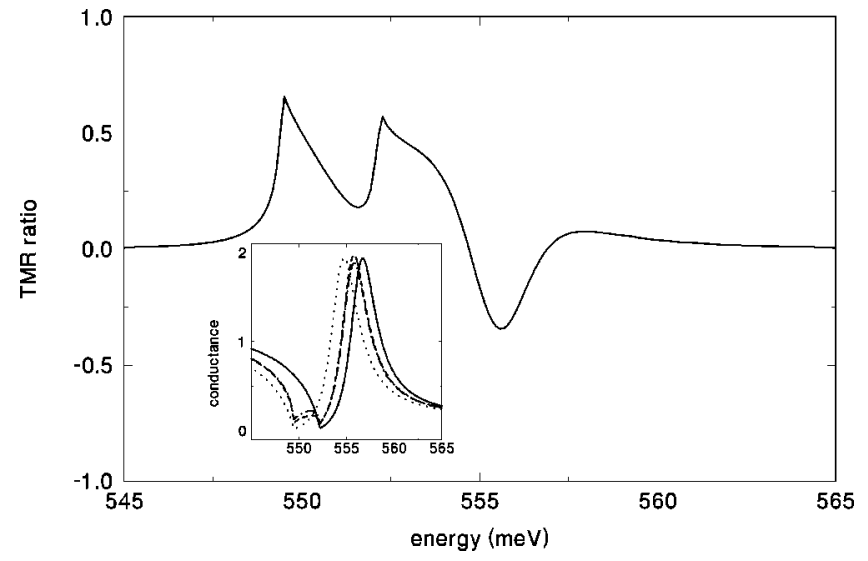

FIG. 4. The TMR ratio vs energy. The external magnetic field is $5 \mathrm{~T}$ and $\mu=5 \mu_{B}$. Inset: the conductances $G_{\uparrow \uparrow}$ (solid line), $G_{\downarrow \downarrow}$ (dotted line), $G_{\uparrow \downarrow}$ (dashed line), and $G_{\downarrow \uparrow}$ (dash-dotted line) vs energy.

increases. It then drops to zero at around $E=550 \mathrm{meV}$, and exhibits a sharp peak at $E=556 \mathrm{meV}$ and a broad peak near $E=720 \mathrm{meV}$. Upon further increasing the energy, the transmission finally cuts off when $E>728 \mathrm{meV}$.

We now examine the behavior of the transmission near the cutoff energy (see the right inset of Fig. 2). We observe that there are two dominating transmission eigen-channels that go to zero near the cutoff one by one $\left[T_{1}=0\right.$ at $E$ $=727.7 \mathrm{meV}$ (dotted line) and $T_{2}=0$ at $E=728.3 \mathrm{meV}$ (dashed line)] resulting to the total transmission coefficient shown in the right inset of Fig. 2 (solid line). The origin of the cutoff energy $E_{c}$ is that when $E>E_{c}$, the scattering wave function in the lead is orthogonal to the wave function of the carbon atoms in the device scattering region; i.e., all three conducting channels are therefore closed. When the magnetic field is switched on (see Fig. 2), conductance $G_{F}$ shows three plateau-like structures, while $G_{A}$ has two. As a result, the TMR ratio exhibits a step-like structure with the largest ratio $\kappa=1$. The physics can be understood as follows. For an electron with a given Fermi energy, the potential landscape of the left (or right) lead seen by the spin-up electron is $U-\mu B$, while for the spin-down electron it is $U+\mu B$. Therefore, due to the change of the potential landscape of a particular lead, the cutoff energy $E_{c}$ will be shifted by $\pm \mu B$ depending on the spin of the electron. As a result, when magnetic fields of the two leads are parallel, the cutoff energy for the transmission coefficient is shifted by an amount $\pm \mu B$ depending on the direction of the spin. If the magnetic fields of two leads are anti-parallel, however, the cutoff energy is shifted down by $\mu B$. In the left inset of Fig. 2, we plot the conductances $G_{\sigma \sigma}$ and $G_{\sigma \bar{\sigma}}$; i.e., when the magnetic fields of two leads are parallel and anti-parallel, respectively. The numerical data are consistent with the above physical argument that the cutoff energy is shifted down by the anti-parallel field. Finally, because $G_{F}$ and $G_{A}$ have different cutoff energies, they behave differently. In Fig. 3, we depict $G_{F}, G_{A}$, and the TMR ratio for $\mu=5 \mu_{B}{ }^{28}$ close to the cutoff energy. Similar results are observed except that the energy window for $\kappa=1$ is now much larger. Indeed, we found that the spin valve effect is rather generic for the system $\mathrm{Al}-C_{4}-\mathrm{Al}$, it occurs at a wide range of parameters. 
Figure 4 shows another interesting behavior of the molecular scale TMR. Near $E=556 \mathrm{meV}$, the transmission coefficient has a large resonant peak as shown in the inset. Near this resonance, we found that both a positive and a negative TMR ratios can be observed. This is because when the magnetic field is turned on, positions of the resonant peak for $\theta$ $=\pi$ remain unchanged, but for $\theta=0$ the peak shifts upward for spin up electron and downward for spin down electron (see inset of Fig. 4), giving rise to a negative TMR ratio.

In summary, we have reported a theoretical formalism for calculating molecular scale spin valves within the NEGFDFT technique. ${ }^{22}$ Application to a carbon atomic chain showed several interesting TMR behaviors. Due to the orthogonalization of the wave function of the atomic lead and the atomic clusters in the device scattering region, there ex- ists a cutoff energy $E_{c}$ for the conductance beyond which the conductance vanishes. In the presence of a magnetic field, electrons with different spins experience different potential landscapes due to the Zeeman effect, which leads to the shifting of the cutoff energy. As a result, the reversal of the magnetic field of one lead can completely switch off the electric current, giving rise to a large TMR ratio of order 1.

\section{ACKNOWLEDGMENTS}

We gratefully acknowledge support by a RGC grant from the HKSAR under grant number HKU 7113/02P, the grant from NSFC under grant number 10274052 (Y.D.W.), and from NSERC of Canada and FCAR of Quebec (H.G).
*Electronic mail: jianwang @ hkusub.hku.hk

${ }^{1}$ G.A. Prinz, Science 282, 1660 (1998); A. Wolf et al., ibid. 294, 1488 (2001).

${ }^{2}$ J.C. Slonczewski, Phys. Rev. B 39, 6995 (1989).

${ }^{3}$ M. Julliere, Phys. Lett. 54A, 225 (1975).

${ }^{4}$ J.S. Moodera, J. Nowak, and R.J. M. van de Veerdonk, Phys. Rev. Lett. 80, 2941 (1998).

${ }^{5}$ K. Tsukagoshi, B.W. Alphenaar, and H. Ago, Nature (London) 401, 572 (1999).

${ }^{6}$ X.D. Zhang, B.Z. Li, G. Sun, and F.C. Pu, Phys. Rev. B 56, 5484 (1997).

${ }^{7}$ A.M. Bratkovsky, Phys. Rev. B 56, 2344 (1997).

${ }^{8}$ S. Zhang, P.M. Levy, A.C. Marley, and S.S.P. Parkin, Phys. Rev. Lett. 79, 3744 (1997).

${ }^{9}$ J. Barnaś and A. Fert, Phys. Rev. Lett. 80, 1058 (1998).

${ }^{10}$ A. Brataas, Yu.V. Nazarov, J. Inoue, and G.E.W. Bauer, Phys. Rev. B 59, 93 (1999).

${ }^{11}$ L. Sheng, Y. Chen, H.Y. Teng, and C.S. Ting, Phys. Rev. B 59, 480 (1999)

${ }^{12}$ H. Mehrez, J. Taylor, H. Guo, J. Wang, and C. Roland, Phys. Rev. Lett. 84, 2682 (2000).

${ }^{13}$ B.G. Wang, J. Wang, and H. Guo, J. Phys. Soc. Jpn. 70, 2645 (2001).

${ }^{14}$ N. Sergueev, Q.F. Sun, H. Guo, B.G. Wang, and J. Wang, Phys. Rev. B 65, 165303 (2002).

${ }^{15}$ F. Mireles and G. Kirczenow, Phys. Rev. B 66, 214415 (2002).

${ }^{16}$ B. Zhao et al., Appl. Phys. Lett. 80, 3144 (2002).

${ }^{17}$ S. Krompiewski, R. Gutiérrez, and G. Cuniberti, Phys. Rev. B 69, 155423 (2004).

${ }^{18}$ L. Wang, J.J. Qiu, W.J. McMahon, K.B. Li, and Y.H. Wu, Phys.
Rev. B 69, 214402 (2004).

${ }^{19}$ For a recent review, see, for example, M.A. Ratner, Mater. Today 5, 20 (2002).

${ }^{20}$ N.D. Lang and Ph. Avouris, Phys. Rev. Lett. 81, 3515 (1998); Phys. Rev. Lett. 84, 358 (2000).

${ }^{21}$ B. Larade, J. Taylor, H. Mehrez, and H. Guo, Phys. Rev. B 64, 075420 (2001).

${ }^{22}$ The NEGF-DFT package MCDCAL is discussed in J. Taylor, H. Guo, and J. Wang, Phys. Rev. B 63, 245407 (2001); Phys. Rev. B 63, 121104(R) (2001).

${ }^{23}$ M. Brandbyge, J.-L. Mozos, P. Ordejón, J. Taylor, and K. Stokbro, Phys. Rev. B 65, 165401 (2002).

${ }^{24}$ D.R. Hamann, M. Schlüter, and C. Chiang, Phys. Rev. Lett. 43, 1494 (1982).

${ }^{25}$ In our calculations, an $\mathrm{Al}$ electrode is composed of unit cells with nine $\mathrm{Al}$ atoms oriented in the (100) direction repeated to $\pm \infty$. The scattering region contains four layers of the $\mathrm{Al}$ atoms on either side of the molecule. The carbon atoms in contact with the electrodes are positioned at the hollow site for $\mathrm{Al}$ electrodes. We fixed the contact distance to be $d=2.0 \AA$. The separation between carbon atoms is $1.3225 \AA$ (see Ref. 27).

${ }^{26}$ It would be better to use the lead made of transition metal rather than Al lead.

${ }^{27}$ H.J. Choi, J. Ihm, S.G. Louie, and M.L. Cohen, Phys. Rev. Lett. 84, 2917 (2000).

${ }^{28}$ For carbon nanotubes, the orbital magnetic moment in the presence of an axial magnetic field can be 10 or 20 times of Bohr magneton (see Ref. 29).

${ }^{29}$ E.D. Minot, Y. Yaish, V. Sazonova, and P.L. McEuen, Nature (London) 428, 536 (2004). 